\title{
A Comparative Study on the Efficacy of Some Artemisinin Combination Therapies on Plasmodium berghei in Swiss Albino Mice
}

\author{
Udeme O. Georgewill*, Omotayo O. Ebong
}

Department of Pharmacology \& Toxicology, Faculty of Basic Medical Sciences, University of Port Harcourt, Choba, Nigeria. Email: *udgeorgewill@yahoo.com

Received October $18^{\text {th }}, 2011$; revised November $20^{\text {th }}, 2011$; accepted December $26^{\text {th }}, 2011$

\begin{abstract}
This study evaluated and compared the efficacy of five brands of Artemisinin Combination Therapies (ACTs); Dihydroartemisinin plus Piperaquine, Artesunate plus Amodiaquine, Artesunate plus Sulphadoxine/Pyrimethamine, Artemether plus lumefantrine and Artesunate plus mefloquine combinations in vivo in P. berghei infected swiss albino mice. The experimental animals were pre-screened to rule out infection. All drugs were administered as clinical doses for the curative test and the Mean Percentage Parasitemia level assessed daily for seven days and on day 60. The results showed that all the drugs were effective with artesunate plus amodiaquine combination being the most efficacious followed by dihydroartemisinin plus piperaquine and artesunate plus sulphadoxine plus pyrimethamine combinations followed by artesunate plus mefloquine combination and artemether plus lumefantrine combination which was the least efficacious. Results on day 60 showed increasing parasitemia levels in mice which received Artemether plus lumefantrine and Artesunate plus mefloquine combinations which is indicative of recrudescence. The results of this study showed that the ACT's used in the experiment were all efficacious. The possible development of resistance to some of the drugs was shown by the increasing parasitemia levels following treatment with artesunate plus lumefantrine and artesunate plus mefloquine combinations on day 60 .
\end{abstract}

Keywords: Efficacy; Malaria; Artemisinin Combination Therapies; Recrudescence; Resistance

\section{Introduction}

Malaria is caused by the parasite plasmodium, which is transmitted by the bite of infected mosquitoes. It is perhaps the world's most devastating human parasitic infection [1].

According to Global Malaria Action Plan, malaria affects 109 countries. However 35 countries are responseble for $98 \%$ of the global malaria deaths. Malaria is a disease of sub-saharan Africa as 30 of the 35 countries are in this region and only 5 of the 30 countries-Nigeria, Democratic Republic of Congo, Uganda, Ethiopia and Tanzania account for $50 \%$ of the global malarial deaths and $47 \%$ of all malarial cases [2]. Since the 1940's, chloroquine has been the drug of choice for both treatment and chemoprophylaxis of malaria but its efficacy against $P$. falciparum has been seriously compromised by drug resistance. In 1946, Amodiaquine was discovered, Primaquine in 1950, and Pyrimethamine in 1952 [3]. In time, Fansidar a fixed combination of the sul-

\footnotetext{
"Corresponding author.
}

phonamide/sulfadoxine and pyrimethamine, was developed. Newer drugs that followed include: halofantrine, lumefantrine, artemisinin and its analogs. In a bid to curb the disease, the World Health Organization strongly recommends ACT regimens as treatment for uncomplicated $P$. falciparum malaria cases in endemic areas [4].

The WHO currently recommends the following combination therapies in Africa;

1) Artemether/lumefantrine

2) Artesunate plus amodiaquine (in areas where the cure rate of amodiaquine monotherapy is $>80 \%$ )

3) Artesunate plus mefloquine

4) Artesunate plus sulfadoxine/pyrimethamine (in areas where the cure rate of $\mathrm{SP}$ is $>80 \%$ )

N/B amodiaquine plus sulfadoxine/pyrimethamine may be considered an interim option where ACTs cannot be made available, provided efficacy of both is high [5].

There are fears among researchers and the Roll Back Malaria partners that there may be a recurrence of epidemics of malaria which occurred in 1950's, as the malaria parasite has developed resistance to the new anti- 
malarial drug artemisinin. In addition, the WHO has warned that resistance to the world's most effective drug against malaria, made out of a combination of drugs, known mostly as the artemisinin therapy, had spread across Asia and had the potential to spread to Africa, putting millions of lives at risk of dying from malaria [6]. This study therefore seeks to evaluate the efficacy of some ACT's and the possible development of recrudescence in mice.

\section{Methodology}

35 Male Swiss albino mice (average weight $28.5 \mathrm{~kg}$ ) obtained from the University of Port Harcourt Animal House was used for this study. The mice were bred and maintained under suitable conditions, housed in hygienic cages in groups of five, fed with growers mash from Top Feeds, Benin City and given clean drinking water ad libitum. The donor mice infected with the chloroquine sensitive Plasmodium berghei was obtained from the National Institute for Medical Research, Yaba, Lagos. The animals were monitored regularly for signs of infection such as shivering, ruffled appearance, lethargy, and anorexia. Level of parasitemia was monitored using the white blood cell count method to determine the number of parasites in a microlitre of blood. Parasites were maintained through weekly passaging of blood to uninfected mice. Blood obtained from donor mice was diluted with phosphate buffered salinesuch that $0.2 \mathrm{ml}$ of diluted blood contained $1 \times 10^{7}$ Plasmodium berghei parasitized red cells which was then injected into each mouse [7].

The mice were placed in seven groups of five mice each for the experiment and all drugs were administered orally. Drugs were administered using dosage regimens as developed [8,9].

1) Group one mice received Dihydroartemisinin(DHA)/ Piperaquine Phosphate (DP) combination (40 mg/320 $\mathrm{mg}$ ). Clinical dose of the drug was given orally as 6.4 $\mathrm{mg} / \mathrm{kg}$ of DHA and $51.2 \mathrm{mg} / \mathrm{kg}$ of piperaquine phosphate at $0,6,24$ and 32 hours.

2) Group two mice received Artemether/Lumefantrine (A/L) combination $(20 \mathrm{mg} / 120 \mathrm{mg})$. Clinical dose administered orally as $1.5 \mathrm{mg} / \mathrm{kg}$ of artemether and $9 \mathrm{mg} / \mathrm{kg}$ of Lumefantrine for six doses given at 0, 8, 24, 3648 and $60 \mathrm{hrs}$

3) Group three mice received artesunate and sulphadoxine + pyrimethamine (AS $+\mathrm{S} / \mathrm{P}$ ) combination (200 $\mathrm{mg} / 500 \mathrm{mg} / 25 \mathrm{mg}$ ) administered as Artesunate $4 \mathrm{mg} / \mathrm{kg}$ once daily for 3 days and SP single dose of $25 \mathrm{mg} / \mathrm{kg}$ and $1.25 \mathrm{mg} / \mathrm{kg}$ respectively.

4) Group four mice received artesunate/amodiaquine (AS + AQ)combination (200/600 mg) administered orally as Artesunate $4 \mathrm{mg} / \mathrm{kg}$ and Amodiaquine $10 \mathrm{mg} / \mathrm{kg}$ once daily for 3 days.

5) Group five mice received artesunate/mefloquine (AS + MQ) combination (600/750 mg) administered orally as Artesunate $4 \mathrm{mg} / \mathrm{kg}$ once daily for 3 days + mefloquine $25 \mathrm{mg} / \mathrm{kg}$ base as a split dose of $8 \mathrm{mg} / \mathrm{kg}$ daily for 3 days.

6) Group six mice received chloroquine tabs (5 mg/kg) twice daily for 3 days.

7) Group seven mice received distilled water regularly

Tail blood collected from treated mice was used for thick and thin films. Parasitemia level was estimated by counting the number of infected cells using a $10 \times 10$ grid square. Several fields were counted and an average taken to represent total parasitemia.

\section{Statistical Analysis}

All results obtained were statistically analyzed and expressed as percentages and as Mean \pm SEM; and the significance of differences of the different drug treated groups were determined using ANOVA. Values of $\mathrm{P}<$ 0.05 were considered as significant.

\section{Results}

KEY: DP = Dihydroartemisinin/piperaquine phosphate combination; $\mathrm{A} / \mathrm{L}=$ Artemether/lumefantrine combination; AS $+\mathrm{S} / \mathrm{P}=$ Artesunate/sulphadoxine + pyrimethamine combination; AS + AQ = Artesunate/Amodiaquine combination; AS $+\mathrm{MQ}=$ Artesunate/Mefloquine combination; $\mathrm{CQ}=$ Chloroquine.

\section{Discussion}

The Artemisinin Combination Therapies (ACTs) used in this study were all efficacious with Mean Percentage Parasitemia (MPP) reduction ranging from $85.71 \%$ to $100 \%$. AS + AQ combination was found to be most effective in treated mice. This was followed by DP and AS $+\mathrm{S} / \mathrm{P}$ with complete parasite clearance by day 7 of the study. AS + MQ came next with an incomplete but reduced parasite clearance of $13 \%$ and $14.29 \%$ parasite clearance following $\mathrm{A} / \mathrm{L}$ administration by day 7 of the study. Further analysis of data shows thus; in order of decreasing efficacy, artesunate + amodiaquine $>$ dihydroartemisinin + piperaquine $=$ artesunate + sulphadoxine/pyrimethamine $>$ artesunate + mefloquine $>$ artesunate + lumefantrine. i.e. $\mathrm{AS}+\mathrm{AQ}>\mathrm{DP}=\mathrm{AS}+\mathrm{S} / \mathrm{P}>\mathrm{AS}+$ MQ > AL Figure 1.

Day 60 results showed that with Artemether plus Lumefantrine and Artesunate plus Mefloquine combinations, there was recrudescence as incompletely cleared parasites multiplied in vivo to as much as $360 \%$ and $250.4 \%$ respectively. i.e. Increase in mean percentage 
parasitemia levels on day 60 following administration of ACTs was in the order of $\mathrm{AL}>\mathrm{AS}+\mathrm{MQ}>\mathrm{DP}=\mathrm{AS}+$ $\mathrm{S} / \mathrm{P}=\mathrm{AS}+\mathrm{AQ}$ Figure 2.

Following administration of DP to P. berghei infected mice showed the drug to be effective in mice with MPP reduction of $100 \%$ by day 7 of the study following treatment. The parasites were totally cleared by day 7 of the study with no recrudescence recorded on day 60 . DP was equi-efficacious with AS $+\mathrm{S} / \mathrm{P}$ combination. This result is consistent with a WHO report that across Asia, Africa, and South America, clinical and parasitological responses to DP have generally exceeded the $95 \%$ value that WHO recommends for antimalarial treatments [10]. Recent studies have confirmed the excellent clinical efficacy of DP combinations (28 - 63 day cure rates greater than 95\%) and have demonstrated that currently recommended regimens are not associated with significant cardiotoxicity or other adverse effects [11].

MPP reduction following treatment of $P$. berghei infected mice with $\mathrm{A} / \mathrm{L}$ was $85.71 \%$ on day 7 . By day 6 , there was no further decrease in parasite level and by day 60 there was an increase of MPP to $360 \%$ indicative of

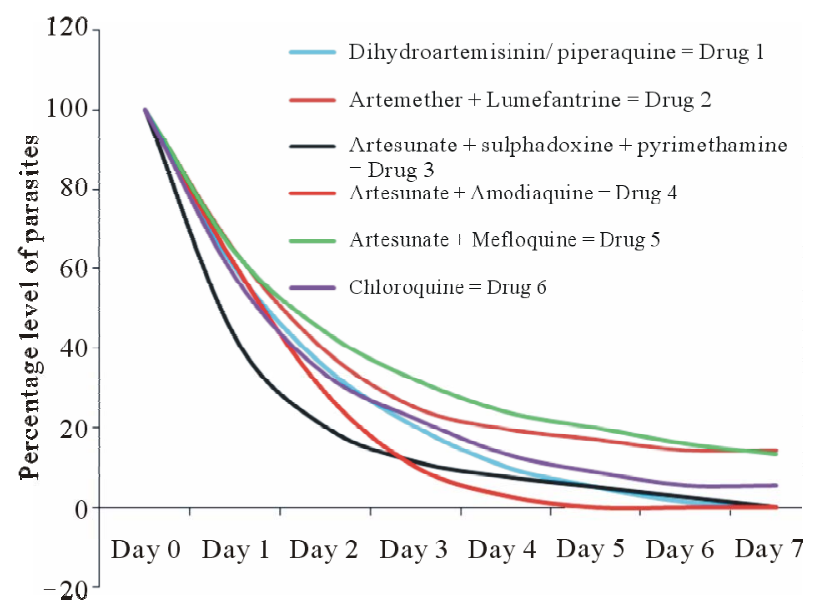

Figure 1. Daily mean percentage parasitemia levels in mice after administration of artemisinin combination therapies.

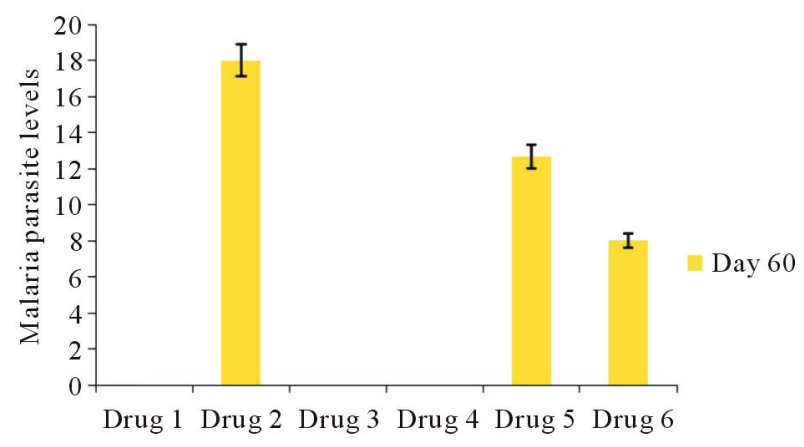

Figure 2. Mean parasite levels in mice on day 60 after treatment with different artemisinin combination therapies. massive recrudescence. By day 7 of the study post treatment, MPP level was $14.29 \%$. This was the least efficacious of the ACTs under study. Clinical studies in Uganda in which the efficacy and safety of DP and AL were compared showed DP was superior to A/L for reducing the risk of recurrent parasitemia and gametocytecemia [12]

AS $+\mathrm{S} / \mathrm{P}$ administration to $P$. berghei infected mice was effective with MPP reduction of $100 \%$ by day 7 . By day 7 , recorded percentage parasite level was $0 \%$. This indicates that the parasites were totally cleared from treated mice. AS $+\mathrm{S} / \mathrm{P}$ was equi-efficacious with DP in this study. Clinical studies in Peru and the Gambia where resistance to Sulphadoxine/Pyrimethamine is uncommon show an efficacy of $100 \%$ [13]. This is consistent with results of this study:

AS + AQ combination was the most effective ACT in treated mice as it cleared the parasite level completely by day 5 of the study. That is; MPP level was $100 \%$ by day 5 of the study, following treatment with total clearance of parasite in treated mice. Animals remained aparasitemic on day 60 with no recrudescence recorded. Literature from Kenya, Senegal, and Gabon where clinical studies on the therapeutic efficacy of artesunate/amodiaquine combination were carried out, reported Day 14 cure rates of $91 \%, 93 \%$ and $98 \%$ which is consistent with the 7-day 100\% MPP levels obtained in the present study [14].

Results of AS + MQ administration recorded a MPP reduction of $85.71 \%$. MPP at day 7 was $13 \%$ with no further reduction but rather a $250.4 \%$ increase in Mean parasite levels by day 60 of the study indicative of a high level of recrudescence. This treatment option may not be suitable for Nigeria and agrees with the Roll Back Malaria technical report that combinations with mefloquine are not recommended for Africa because of the effect that mefloquine pressure has had on the selection of resistant parasites in areas of intense transmission [15].

We therefore state that from the present study in mice, the Acts studied were all efficacious and that there might be development of resistance to some of the artemisinin based combination therapies and we advocate that further studies be carried out to assess the safety profile of these drugs in mice and in vitro studies in humans to assess the efficacy of these drugs.

\section{Acknowledgements}

The authors are grateful to Mr. Joshua Isirimah and Mrs Mathilda Deekor for their technical assistance.

\section{REFERENCES}

[1] J. W. Nicholas and J. G. Breman, "Malaria and Babeiosis; Diseases Caused by Red Blood Cell Parasites in Harri- 
sons Principle of Medicine,” McGraw Hill, New York, 2005, p. 1218.

[2] N. Odebode and H. Shobiye, "Malaria Epidemics Loom as Parasites Develops Resistance to New Drug," An Article in the Punch Newspapers Dated 12 July 2010. http://www.punch ng.com

[3] R. Ramachandra, "Resistance to Antimalarial Drugs Frontline,” Health (Indians National Magazine), 2004, pp. 34-37.

[4] S. Ehrhardt and C. G. Meyer, "Artemether-Lumefantrine in the Treatment of Uncomplicated Plasmodium Falciparum Malaria,” Therapeutics and Clinical Risk Management, Vol. 5, 2009, pp. 805-815. doi:10.2147/TCRM.S5375

[5] WHO Report, "Roll Back Malaria Partnership; Facts on ACTs (Artemisinin Based Combination Therapies," January 2006 Update. http://www.rollbackmalaria.org

[6] Afrique en ligne, "Malaria Drug Resistance Warning Puts African Health Researchers on Edge,” Africa News, 2010. http://www.afriquejet.com

[7] U. Rane and P. Sehdeu, "Malaria Drug Development," 1993. http://www.aaas.org/international/Africa/malaria/rane.ht $\mathrm{ml}$

[8] E. A. Ashley, K. Stepniewska and N. Lindegårdh, "Pharmacokinetic Study of Artemether-Lumefantrine Given Once Daily for the Treatment of Uncomplicated Multidrugresistant Falciparum Malaria,” Tropical Medicine and International Health, Vol. 12, No. 2, 2007, pp. 201-208. doi:10.1111/j.1365-3156.2006.01785.x

[9] B. S. Kakkilaya, “Combinatios of Antimalarial Drugs,”
Dr B.S. Kakkilaya’s Malaria Website, 2009.

[10] WHO Report, “World Malaria Report,” 2008. http://www.who.int/malaria/wmr

[11] H. Myint, E. Ashley, N. Day, F. Nosten and N. White, "Efficacy and Safety of Dihydroartemisinin-Piperaquine," Tropical Medicine and Hygiene, Vol. 101, No. 9, 2007, pp. 858-866. doi:10.1016/j.trstmh.2007.05.018

[12] M. R. Kamya, A. Yeka, H. Bukirwa, M. Lugemwa and J. B. Rwakimari, “Artemether-Lumefantrine versus Dihydrortemisinin-Piperaquine for Treatment of Malaria: A Randomized Trial,” PLoS Clinical Trials, Vol. 2, No. 5, 2007, p. e20.

[13] M. Wilmer, Y. Laura, H. Ygor, A. Palacios, F. Eduardo, C. Cabezas, N. Arrospide, S. Guttierez and K. Trenton, "Efficacy and Tolerability of Artesunate plus Sulfadoxine-Pyrimethamine and Sulfadoxine Pyrimethamine Alone for the Treatment of Uncomplicated Plasmodium Falciparum Malaria in Peru," American Journal of Tropical Medicine and Hygiene, Vol. 72, No. 5, 2005, pp. 568-572.

[14] M. Meremikwu, A. Alaribe, R. Ejemot, A. Oyo-ita, J. Ekenjoku C. Nwachukwu, D. Ordu and E. Ezedinachi, "Artemether/Lumefantrine versus Artesunate plus Amodiaquine for Treating Uncomplicated Childhood Malaria in Nigeria: Randomized Controlled Trial,” Malaria Journal, Vol. 5, No. 43, 2006, PMC Free Article. doi:10.1186/1475-2875-5-43

[15] P. Olumese, “Antimalarial Combination Therapy,” Capacity Development and Technical Support, Roll Back Malaria, 2009. http://www.apps.who.inf/malaria 